\title{
Title
}

\section{Functional characterization of two variants in the mitochondrial topoisomerase gene TOP1MT that impact regulation of the mitochondrial genome}

\section{Authors and Affiliations}

Iman Al Khatib(1), Marina Kerr(2), Hongliang Zhang(3), Sharyin Huang(3), Yves Pommier(3), Aneal Khan(2,4), Timothy E Shutt(5)*

1) Department of Biochemistry \& Molecular Biology, Cumming School of Medicine, University of Calgary, Calgary, AB, Canada; Alberta Children's Hospital Research Institute, University of Calgary, Calgary, Alberta, T2N 4N1, Canada.

2) Discovery DNA, Calgary, Alberta, T2L 1Y8, Canada

3) Laboratory of Molecular Pharmacology, Developmental Therapeutics Branch, Center for Cancer Research, $\mathrm{NCl}$, National Institutes of Health, Bethesda, MD, 20892, USA.

4) M.A.G.I.C. Clinic Ltd. (Metabolics and Genetics in Calgary); Department of Pediatrics, Cumming School of Medicine, University of Calgary, Calgary, AB, Canada; Alberta Children's Hospital Research Institute, , Calgary, Alberta, T2M OL6, University of Calgary Canada.

5) Department of Biochemistry \& Molecular Biology, Cumming School of Medicine, University of Calgary, Calgary, AB, Canada; Department of Medical Genetics, Cumming School of Medicine, University of Calgary, Calgary, AB, Canada; Alberta Children's Hospital Research Institute, University of Calgary, Calgary, Alberta, Canada; Hotchkiss Brain Institute, University of Calgary, Calgary, Alberta, T2N 4N1, Canada.

*Corresponding Author: timothy.shutt@ucalgary.ca 


\section{Abstract}

TOP1MT encodes a mitochondrial topoisomerase that is important for mtDNA regulation, and is involved in mitochondrial replication, transcription and translation. Two variants predicted to affect TOP1MT function (R199C and V338L) were identified by exome sequencing of a newborn with hypertrophic cardiomyopathy. As no pathogenic TOP1MT variants have been confirmed previously, we characterized these variants for their ability to rescue several TOP1MT functions in knockout cells. Consistent with a role for these TOP1MT variants contributing to the patient phenotype, comprehensive characterization suggests that both variants had impaired topoisomerase activity and demonstrates that neither variant was able to restore steady state levels of mitochondrial encoded proteins, nor reduced oxidative phosphorylation. However, the two variants behaved differently in some respects. While the $\mathrm{R} 199 \mathrm{C}$ variant was better at restoring transcript levels, the V338L variant was able to restore mtDNA copy number and replication. These findings suggest that the different TOP1MT variants affect distinct TOP1MT functions. Altogether, these findings begin to provide insight into the many roles that TOP1MT plays in the maintenance and expression of the mitochondrial genome, and how impairments in this important protein may lead to human pathology. 


\section{Introduction}

Mitochondria maintain a highly reduced genome (mtDNA) that is evidence of their bacterial ancestry. In humans, the circular $\sim 16.5 \mathrm{~kb}$ genome encodes 13 proteins that are essential components of oxidative phosphorylation (OxPhos) complexes, as well as two ribosomal RNAs and 22 tRNAs required for mitochondrial translation. As such, replication, maintenance, and expression of mtDNA is important for many mitochondrial functions. Mitochondrial genomes are present in hundreds to thousands of copies per cell and are packaged into proteinaceous structures known as nucleoids, which typically each contain one to two copies of the genome[1]. Although we do not have a complete understanding of how mtDNA abundance is maintained, mtDNA copy number is generally thought to reflect the energy production in cells, and mtDNA depletion is linked to human disease[2].

Key regulatory elements for mtDNA replication and transcription are located within an $\sim 1100$ bp non-coding portion of the genome known as the D-loop region, due to the presence of a stable triple-stranded DNA structure that can form[3]. This D-loop comprises a short strand of DNA known as the 7S DNA that is found in a subset of mtDNA molecules, and can be replicated independently of the entire genome. While the D-loop is important for mtDNA, the factors that regulate its biogenesis are poorly understood, and the exact role it plays in regulating mtDNA remains undefined. Meanwhile, expression of the mtDNA is initiated at three different promoters, a light strand promoter (LSP), and two heavy strand promoters (HSP1 and HSP2), which lead to polycistronic transcripts. Notably, LSP also plays an essential role in priming mtDNA replication[4]. Thus, the D-loop region is important to balance replication and expression of the mtDNA genome, though the exact mechanisms remain unknown.

Replication, maintenance, and transcription of mtDNA is performed by proteins that are encoded in the nucleus[5-7]. The fact that mitochondria use a dedicated set of core machinery for mitochondrial transcription[6] and replication[8] highlights the fact that these processes are mechanistically distinct in mitochondria compared to the nucleus. However, there are also several proteins that function in both mitochondrial and nuclear DNA maintenance. Importantly, our understanding of how the mtDNA is regulated is still incomplete.

Topoisomerases cut and re-ligate the DNA backbone to resolve torsional strain, crossovers and knots, are thus important for maintenance of the circular mtDNA genome[9]. To date four of the six human topoisomerases have been found in mitochondria (i.e. TOP1MT, TOP $2 \alpha$, TOP $2 \beta$ and TOP $3 \alpha$ ). However, TOP1MT is the only topoisomerase that is found exclusively in mitochondria[10,11], suggesting a special role for this protein in maintaining mitochondrial function. TOP1MT can relieve negative supercoiling of the mtDNA via its topoisomerase activity, which is thought to be important for its roles in mediating mtDNA replication and transcription [12-14]. TOP1MT also binds mtDNA in the D-loop region, where it is implicated in regulating formation of the 7S DNA[12]. In addition, TOP1MT has a distinct role in mitochondrial translation[15]. Nevertheless, exactly how TOP1MT performs these various roles remains to be fully elucidated.

Here, we characterize two putative pathogenic TOP1MT variants identified in a patient who presented with hypertrophic cardiomyopathy as a newborn infant. While we show that both variants have reduced functionality, which may explain the patient pathology, they also 
have distinct differences that begin to shed light on the roles that TOP1MT plays in mediating mitochondrial function.

\section{Materials and Methods}

\section{Consent}

The methods used to identify the variants through Whole Exome Sequencing (WES) have been described previously in the MITO-FIND pipeline[16], sponsored by MitoCanada (http://mitocanada.org) and in-kind support from Discovery DNA (Calgary, Alberta, Canada). Consent was obtained following standard procedures through the University of Calgary Conjoint Research Ethics Board.

\section{Cell culture}

HCT 116 control, KO and stably transfected cells were cultured in McCoy's 5A (modified) media (Gibco, 16600-82) containing L-Glutamine and supplemented with $10 \%$ fetal bovine serum (FBS). Unless otherwise indicated, cells harvested for analysis were seeded at $1.5 \times 10^{6}$ cells in $10 \mathrm{~cm}$ plates and allowed to grow for two days, reaching approximately $75 \%$ confluence. Phoenix cells used to make retrovirus were grown in DMEM (Gibco, 11965092) supplemented with $10 \%$ FBS. All cells were maintained at $37^{\circ} \mathrm{C}$ and $5 \% \mathrm{CO}_{2}$.

\section{Plasmids \& cloning}

The TOP1MT open reading frame (ORF) was cloned into the pRV100G retroviral vector containing a C-terminal T2A mCherry tag, which encodes an mCherry open reading frame following self-cleaving P2A site. The TOP1MT C592T and G1012T variants were introduced using site-directed mutagenesis. An empty vector containing only mCherry was used as a control.

\section{Generation of stable lines generation}

Stable cells expressing wild type TOP1MT, TOP1MT variants, or empty vector controls were generated via retroviral transduction. Briefly, Phoenix cells were transfected with the retroviral plasmids, and $5 \mathrm{ml}$ of the supernatant containing virus from these cells were used after 48 and $72 \mathrm{hrs}$ to transduce HCT WT or TOP1MT-KO cells at $60 \%$ confluency in $100 \mathrm{~mm}$ plates. Following transduction, approximately $6 \times 10^{6}$ cells were sorted for red fluorescence using a $130 \mu \mathrm{m}$ nozzle on a BD FACSAria Fusion (FACSAriallI) cytometer (BD Biosciences), supported by FACSDiva Version 8.0.1.

\section{PCR and sequencing}

Total DNA was extracted using PureLink Genomic DNA Mini Kit (Thermo Fisher Scientific, K182001). PCR amplification of TOP1MT was used to confirm the success of the transduction using the forward primer CACAACAAAGGAGGTTTTCCGGAAG and the reverse primer TGCAGTCCTTCCCCAGGA. The amplified band was further sequenced using Sanger sequencing to confirm the variant status of TOP1MT in each line.

\section{Live cell imaging}


For live cell imaging, $8 \times 10^{4}$ cells were seeded on $35 \mathrm{~mm}$ glass bottom dishes and grown for two days. To visualize mitochondrial networks and mtDNA nucleoids, cells were simultaneously stained with MitoTracker Red (50 nM) (Thermo Fisher Scientific, M7512) and PicoGreen (Thermo Fisher Scientific, P7581) $(3 \mu \mathrm{L} / \mathrm{mL}$ ) as performed previously[17].

\section{Immunofluorescence staining}

Cells were seeded at $1.5 \times 10^{4}$ cells over $12 \mathrm{~mm}$ glass coverslips (no. 1.5 ) in 24 wells plates and incubated for 2 days. Subsequently, cells were fixed with $4 \%$ paraformaldehyde and stained with mitochondrial networks labeled with a primary antibody against TOMM20 (SigmaAldrich, HPA011562) used at 1:1000, and appropriate Alexa fluor-conjugated secondary antibodies (Thermo Fisher Scientific) at 1:1000.

\section{Microscopy}

Fixed samples were imaged on an Olympus spinning disc confocal system (Olympus SD OSR) (UAPON 100XOTIRF/1.49 oil objective) operated by Metamorph software. A cellVivo incubation module was used to maintain cells at $37^{\circ} \mathrm{C}$ and $5 \% \mathrm{CO} 2$ during live cell imaging.

\section{Image analysis for mtDNA nucleoids}

Nucleoid counts and size measurements were obtained using the particle analysis tool in ImageJ FIJI after binarizing the images[18]. Surface area (size) of each nucleoid and the total nucleoid count per cell were automatically determined for a manually selected region of interest containing the entire mitochondrial network, but excluding the nucleus. The analyses were performed on at least 10 cells for each of the indicated cell lines. For live cells stained with PicoGreen, a violin plot was used to represent the distribution of mtDNA nucleoid sizes and counts for the indicated cell lines. For fixed cells stained with anti-DNA antibody, nucleoid sizes are presented as the average size \pm SEM. The non-parametric Kolmogorov-Smirnov (K-S) test was used to determine statistical significance regarding the distribution of counted nucleoids.

\section{Image analysis for mitochondrial networks}

Mitochondrial network morphology was qualitatively analyzed by classifying networks into three categories, fragmented (predominantly small mitochondrial fragments), intermediate (cells containing a mixture of short fragments and elongated networks) and fused (elongated, interconnected networks with few to no short fragments)[17]. For each cell line investigated, at least 50 cells were scored. Morphology analyses were performed on 3 independent replicates. The results shown represent mean \pm SEM, and $P$ values were based on unpaired, 2-tailed Student's $t$-tests. Average mitochondrial length was measured using ImageJ FIJI. Briefly, background signal was subtracted, images of mitochondrial networks were skeletonized, and the mitochondrial length was obtained using the analyze skeleton function in ImageJ FIJI. The sum of the lengths of all branches of a mitochondrion was evaluated as the total length of that mitochondrion in every image[19]. For each of WT and KO cells, at least 20 cells were evaluated. The results shown are one of three independent biological replicates with the same trends showing mean \pm SEM, and $p$ values based on unpaired, 2-tailed Student's t-tests.

\section{Long-range PCR}


To examine mtDNA deletions, we amplified nearly the full length mtDNA (16.3 kb) as described by Nishigaki et al.[20] using the following primers (1482-1516 F: ACCGCCCGTCACCCTCCTCAAGTATACTTCAAAGG; 1180-1146 R:

ACCGCCAGGTCCTTTGAGTTTTAAGCTGTGGCTCG). Long range PCR reactions were performed using the Takara LA Taq polymerase (Takara Bio, RR002M), with 250 ng genomic DNA, $200 \mathrm{nM}$ forward and reverse primers. The PCR cycling conditions were as follows: $94{ }^{\circ} \mathrm{C}$ for $1 \mathrm{~min} ; 98^{\circ} \mathrm{C}$ for $10 \mathrm{~s}$ and $68^{\circ} \mathrm{C}$ for $11 \mathrm{~min}$ ( $30 \mathrm{cycles}$ ); and a final extension cycle at $72{ }^{\circ} \mathrm{C}$ for $10 \mathrm{~min}$. PCR products were visualized by electrophoresis on a $0.8 \%$ agarose gel, run for approximately $16 \mathrm{~h}$ at $20 \mathrm{~V}$.

\section{mtDNA copy number analysis}

Total DNA was isolated from cultured cells using the PureLink Genomic DNA Mini Kit (Thermo Fisher Scientific, K182001) according to the manufacturer's protocol, and 50-100 ng of DNA was used for quantitative PCR (QPCR) to determine mtDNA copy number, as reported previously[21]. Primers used are shown in Table 1. Briefly, mtDNA or 18S DNA were amplified using PowerUp SYBR Green Master Mix (Thermo Fisher Scientific, A25742) in the QuantStudio 6 Flex Real-Time PCR system (Thermo Fisher Scientific) machine, and the delta delta Ct method was used to determine mtDNA copy number relative to the 18S. Reactions were performed in triplicate and mtDNA copy number analysis was performed on at least three independent biological replicates. Data is presented as mean \pm SEM and unpaired, 2-tailed Student's $t$-tests were used to determine statistical significance.

\section{Ethidium bromide depletion/repletion}

Cells were treated with $1 \mu \mathrm{g} / \mathrm{ml}$ Ethidium bromide (Fisher Scientific, 15-585-011) for three days, as described previously[22], at which point fresh media was added. Cell pellets were collected at days $0,3,6$ and 8, for DNA purification and copy number analysis.

\section{Western}

Cell pellets were collected after trypsinization, washed with $1 \times$ phosphate buffered saline (PBS) and lysed with RIPA buffer (Thermo Scientific ${ }^{\mathrm{TM}}$, 89900) containing protease inhibitors. 20-50 $\mu$ g of total cell lysates were resolved on SDS-PAGE gels and transferred onto PVDF membranes. Blots were probed with the following antibodies at the indicated dilutions; OxPhos antibody cocktail (Abcam, ab110411; 1:500 dilution), anti-Actin (Sigma, A5316; 1:1000), anti-NDUFB6 (Abcam, ab110244; 1:1000), anti-MTCO2 (Abcam, ab110258; 1:1000), anti-GAPDH (Millipore Sigma, ABS16; 1:1000), anti-VDAC1 (Abcam, ab14734; 1:1000), anti-TOP1MT-3 (Developmental Studies Hybridoma Bank, CPTC-TOP1MT-3; 1:200). Appropriate horseradish peroxidase (HRP)-conjugated secondary antibodies were used at 1:3000 as follows: goat anti rabbit IgG, HRP linked Antibody (Cell Signaling Technology, 7074S), or goat anti-mouse IgG-HRP (Santa Cruz Biotechnology, sc-2055). Blots were incubated with Clarity ECL substrate (Biorad, 1705061) and imaged using an Amersham Imager Al600.

\section{Mitochondrial respiration}

Mitochondrial bioenergetic profiles were obtained using a Seahorse XFe24 Extracellular Flux Analyzer (Agilent Technologies, Inc) with the Seahorse XF Cell Mito Stress Test. Briefly, 8 
$\times 10^{4}$ cells/well were seeded in an XF24 microplate and incubated at $37^{\circ} \mathrm{C}, 5 \% \mathrm{CO}_{2}$ for $48 \mathrm{~h}$. Subsequently, growth media was replaced with assay media supplemented with D-Glucose $(25 \mathrm{mM})$, sodium pyruvate $(2 \mathrm{mM})$ and L-Glutamine $(4 \mathrm{mM})$. Oxygen consumption rates were measured following sequential injection of the following compounds into each well: oligomycin $(1 \mu \mathrm{g} / \mathrm{mL})$ (Enzo Life Sciences, BML-CM111), carbonyl cyanide 4-(trifluoromethoxy) phenylhydrazone (FCCP, $1 \mu \mathrm{M}$ ) (Enzo life Sciences, BML-CM120) and Antimycin A (1 $\mu \mathrm{M})$ (Sigma Aldrich, A8674). Upon completion of the assay, protein concentrations from each well were measured by BCA assay (Thermo Fisher Scientific, 23225) and used to normalize data.

\section{RNA extraction and quantification}

RNA was extracted from cell pellets using the E.Z.N.A. ${ }^{\circledR}$ HP Total RNA Kit, Omega Biotek $^{\circledR}$ (VWR Scientific, CA101414-850), according to the manufacturer's protocol. Following extraction, cDNA was generated from $7.5 \mu \mathrm{g}$ of RNA using the iScript ${ }^{\mathrm{TM}}$ Advanced cDNA Synthesis Kit (Biorad, 1725038), and 15-20 ng of cDNA was then used for quantitative PCR with PowerUp SYBR Green Master Mix (Thermo Fisher Scientific, A25742) in the QuantStudio 6 Flex Real-Time PCR system (Thermo Fisher Scientific) machine. The delta delta Ct method was used to determine the relative expression of various transcripts using the primers indicated in Table 1 relative to Actin expression. Reactions were performed in triplicate and mtDNA copy number analysis was performed on at least three independent biological replicates. Data is presented as mean \pm SEM and unpaired, 2-tailed Student's $t$-tests were used to determine statistical significance.

\begin{tabular}{|l|l|l|}
\hline Gene & Forward Primer & Reverse Primer \\
\hline $18 \mathrm{~S}$ & TAGAGGGACAAGTGGCGTTC & CGCTGAGCCAGTCAGTGT \\
\hline mito & CACCCAAGAACAGGGTTTGT & TGGCCATGGGTATGTTGTTAA \\
\hline $12 \mathrm{~S}$ & TAGAGGAGCCTGTTCTGTAATCGAT & CGACCCTTAAGTTTCATAAGGGCTA \\
\hline COX I & GACGTAGACACACGAGCATATTTCA & AGGACATAGTGGAAGTGAGCTACAAC \\
\hline ND 1 & AAGTCACCCTAGCCATCATTCTAC & GCAGGAGTAATCAGAGGTGTTCTT \\
\hline ND 6 & CAAACAATGTTCAACCAGTAACCACTAC & ATATACTACAGCGATGGCTATTGAGGA \\
\hline Cyt B & ATCACTCGAGACGTAAATTATGGCT & TGAACTAGGTCTGTCCCAATGTATG \\
\hline ATP 6 & TAGCCATACACAACACTAAAGGACGA & GGGCATTTTTAATCTTAGAGCGAAA \\
\hline Actin & AAGACCTGTACGCCAACACA & AGTACTTGCGCTCAGGAGGA \\
\hline
\end{tabular}

Table 1: Primers used for quantitative PCR for detection of different genes as indicated where $18 \mathrm{~S}$ and mito were used for detection of mtDNA copy number and the rest for RNA expression of various genes

\section{Results}

\section{Patient Description}

We report a newborn male infant who at birth presented with hypertrophic cardiomyopathy characterized primarily by concentric left ventricular hypertrophy with biventricular hypertrophy and prominent trabeculations. The patient was one of two nonidentical dichorionic/diamniotic twins born to a 50-year-old mother. Both parents were of Nigerian ancestry. Whereas the other twin was healthy, the patient was born with severe hypertrophic obstructive cardiomyopathy. While the mother had a history of gestational diabetes, the cardiac hypertrophy in the patient was severe in nature, and being present in only 
one of the twins, was considered needing further investigations, and prompted testing through WES. Additional clinical workup of the patient at birth showed hyperglycaemia, which could be due to stress of the unusual birth, and hepatomegaly. The acylcarnitine profile showed elevated C18:2, C16 and C18 acyl-carnitine species. However, WES did not identify any variants in genes associated with trifunctional protein deficiency or beta-oxidation that would be considered biologically impactful. There was no elevation in blood lactate or alanine. The cardiac hypertrophy responded to treatment with a beta-blocker, propranolol, and the infant was discharged. The family moved to a different country, and no follow up scans or other samples from the family were available. At a 6-month follow-up contact, the baby was reported to be healthy, but had not seen a cardiologist or had further testing in his area of residence.

\section{Variant Identification}

Whole exome sequencing revealed the patient had compound heterozygous variants in TOP1MT (NM_052963.2). The first variant, V1, was found at position 592C $>\mathrm{T}$, and causes an arginine to leucine change at amino acid 198 (Arg198Cys). The second variant, V2, was found at 1012 G > T, and yields a valine to leucine change at residue 338 (Val338Leu). Parental DNA was not available for segregation testing. Both identified variants occur at highly conserved sites in the core domain of TOP1MT (Fig 1a) and were predicted to be deleterious and damaging with allele frequencies of 0.000527 for V1 and 0.00987 for V2 in Genome Aggregation Database (GnomAD). Notably, the V2 variant was reported previously as a potentially deleterious genomic missense variant for TOP1MT[23]. Nonetheless, as TOP1MT is not formally recognized as a human disease gene, additional functional characterization of these TOP1MT variants was required to investigate their potential pathogenicity.

\section{Cell Line Generation}

To examine the functionality of the V1 and V2 variants, we wanted to characterize the ability of these variants to rescue functions when re-expressed in an HCT116 cell line where TOP1MT was knocked via CRISPR-Cas9[22]. Starting from HCT116 wild type or HCT116 TOP1MT KO cells (labelled HCT-WT or HCT-KO, respectively), we generated five different stable cell lines using retroviral constructs for TOP1MT-P2A-mCherry, or an empty vector control. As controls to eliminate the effect of retroviral transduction and mCherry expression, the HCT-WT and HCTKO cells were transduced with the P2A-mCherry empty vector (noted as WT-ctrl and KO-ctrl). Importantly, the empty vector WT-ctrl and KO-ctrl lines behaved similar to their untransduced parental HCT-WT or HCT-KO lines with respect to the TOP1MT functions investigated throughout this paper (Supplemental Figure S1), indicating that the viral transduction itself had no major impact. Next, to examine the rescue effects of re-expressing wild type TOP1MT in KO cells, a third cell line was generated by re-expressing the wild type form of TOP1MT (indicated as Rescue). Finally, to evaluate the ability of the patient variants to rescue loss of TOP1MT function in KO cells, two additional cell lines expressing either the V1 or V2 variants were established (referred to as V1 or V2, respectively).

Viral transduction of cells was confirmed by visualizing mCherry expression via confocal microscopy and cells expressing mCherry were sorted by flow cytometry to select the population of transduced cells. The transduction of WT, V1 and V2 TOP1MT was further confirmed by PCR amplification of the TOP1MT open reading frame (Fig 1b), and the variant 
status of the Rescue, V1 and V2 lines was confirmed by DNA sequencing (Fig 1c). Next, we confirmed the expression of TOP1MT proteins by immunoblotting (Fig 1d). A band of the predicted size of $\sim 70 \mathrm{kDa}$ corresponding to TOP1MT protein was observed in HCT-WT and WTctrl cells, which is missing from the HCT-KO and KO-ctrl cells. A band migrating at the same size was observed in the Rescue, V1, and V2 lines (Fig 1e). Immunofluorescence imaging confirmed the protein expression findings from the western blotting, and also showed that WT, V1, and V2 TOP1MT proteins are indeed localized to mitochondria (Fig 2). Having established these cell lines, we then wanted to determine whether phenotypic differences in KO-ctrl cells compared to WT-ctrl cells (Supplemental Figure S1) could be rescued by re-expression of WT, V1, or V2 TOP1MT variants. Thus, we examined the several cellular parameters.

\section{Nucleoids}

Given the role of TOP1MT as a mitochondrial DNA topoisomerase that reverses negative supercoiling of mtDNA[24], we examined the effect of TOP1MT deletion on nucleoids. We employed confocal microscopy immunofluorescence to visualize nucleoids in live cells labelled with picogreen (Fig 3a). Although there was a trend towards an increase in the average number of nucleoids per cell in the KO cells, this was not statistically significant (Fig 3b). However, KO cells did show a significant $\sim 30 \%$ reduction in nucleoid size, consistent with increased negative supercoiling (Fig 3c). Similar results were obtained in fixed cells labelled with an anti-DNA antibody (Supplemental Figure S2). The change in nucleoid size was not due to the accumulation of large mtDNA deletions, as long-range PCR confirmed that there were no mtDNA deletions in either the HCT ctrl or HCT-KO cell lines (Fig 3d). Importantly, a control cell line bearing a known $5 \mathrm{~kb}$ deletion was used as a positive control to show the assay can detect mtDNA deletions. We next asked whether the reduced nucleoid size in the HCT-KO cells could be rescued by re-expressing TOP1MT. Critically, overexpression of the WT TOP1MT protein reversed the nucleoid size phenotype, whereas the overexpression of V1 and V2 variants led to intermediate nucleoid sizes, with V1 showing better rescue compared to V2 (Fig 4a\&b).

\section{mtDNA copy number and replication}

Although the average number of nucleoids/cell quantified by imaging was not significantly different, previous reports have shown changes in mtDNA copy number under certain conditions of TOP1MT impairment[22]. Thus, we employed quantitative PCR as a more sensitive assay to look at mtDNA copy number. We found that mtDNA copy number was significantly elevated by $\sim 40 \%$ in HCT-KO cells compared to HCT-WT cells (Fig 5a), and that this was corrected in the Rescue cells re-expressing WT TOP1MT. Moreover, while expression of V1 did not restore the copy number, expression of $\mathrm{V} 2$ did provide rescue.

In addition to examining steady state mtDNA levels, we also examined the ability of the mtDNA to replicate. A common assay for mtDNA replication is a depletion/repletion assay, whereby the intercalating agent ethidium bromide blocks mtDNA replication leading to copy number depletion[25, 26]. Subsequent removal of ethidium bromide allows mtDNA replication such that repletion of mtDNA copy number can be measured[27]. Similar to previous reports[22, 28], we found that mtDNA repletion in KO-ctrl cells is significantly reduced compared to WT-ctrl (Fig 5b). Meanwhile, although re-expression of WT TOP1MT increased repletion, above levels of even the WT cells, the V1 and V2 variants had different effects. The 
V1 variant showed no increase in repletion, while the V2 variant showed improved repletion, similar to WT-ctrl cells, but still reduced compared to the Rescue cells.

\section{Mitochondrial Transcription}

As transcription of the mtDNA genome is intricately linked to mtDNA replication and is also influenced by mtDNA supercoiling mediated by TOP1MT[13, 14], we examined the steadystate levels of mtDNA transcripts. Unexpectedly, despite the finding that mtDNA copy number was higher in HCT-KO cells, mtDNA transcripts from LSP and HSP2 promoters were significantly lower in these cells compared to HCT-WT (Fig 5c). Meanwhile, levels of the rRNA transcripts from HSP1 were not affected.

As the reduced transcript levels that we observed contradicted previous reports showing increased levels of mtDNA-encoded transcripts in cells lacking TOP1MT[14, 24, 29, 30], we examined the relative transcript levels in cells grown at different seeding densities (Supplemental Fig S3). We found that at lower seeding densities, similar to the experimental conditions used in this paper, HCT-KO cells had reduced levels of mtDNA-encoded transcripts. Conversely, at higher seeding densities where cells were confluent upon harvesting for experimentation, HCT-KO cells had increased levels of mtDNA-encoded transcripts.

Importantly, transcript levels for HSP2 and LSP were restored to normal by reexpressing WT TOP1MT in the Rescue cells (Fig 5d). With respect to the effect of the pathogenic variants, re-expression in the V1 cells was able to rescue both HSP2 and LSP transcript levels. Meanwhile, the V2 cells maintained lower HSP2 transcript levels but did show some rescue for LSP.

\section{Expression of Mitochondrial Proteins}

As TOP1MT can also influence mitochondrial translation, we investigated the expression of OxPhos proteins in our cell lines. We found several intriguing differences when we examined the expression of several subunits of different OxPhos complexes (Fig 6a). Most notably, expression of Complex I subunits NDUFB8 (Fig 6b) and ND6 (Fig 6g), Complex II subunit SDHB (Fig 6c), Complex III subunit UQCRC2 (Fig 6d), complex IV subunit COXII (Fig 6e) and Complex V subunit ATP5A (Fig 6f) showed similar trends with reduced expression in KO-ctrl cells compared to WT-ctrl cells. Although we did not see a significant decrease in UQCRC2 in these experiments, we did observe a decrease in the parent HCT-KO compared to HCT-WT cells (Supplemental Figure S1c). Importantly the expression of all these proteins in the Rescue cell line was restored, sometimes even increased above WT-ctrl levels. Meanwhile, the expression of these proteins in the V1 and V2 lines showed variable amounts of rescue. Generally, expression of OxPhos proteins in the V1 and V2 lines tended to be less than that in the Rescue line. Critically, there was little to no rescue for the mtDNA encoded proteins ND6 and COXII, indicating that these variants could not restore mitochondrial translation.

\section{Mitochondrial Respiration}

Given these changes in levels of OxPhos complex protein expression, we next investigated mitochondrial respiration in our cells (Fig 6h). Similar to previous work[15], we found that both basal respiration (Fig 6i), maximal respiration (Fig 6j), and ATP-coupled respiration (Fig 6k), were all reduced in KO-ctrl cells. While re-expressing of WT TOP1MT in the 
Rescue cells showed restoration of all these parameters, the patient variants had different effects. Expression of V1 provided partial rescue, while the V2 variant provided no rescue at all.

\section{Mitochondrial morphology}

Mitochondrial morphology can change in response to different stresses and is also important for the regulation of mtDNA. Thus, as another measure of mitochondrial fitness, we decided to look at mitochondrial morphology in our stable cell lines using confocal microscopy (Fig 7). We observed a marked increase in the number of cells with hyperfused mitochondrial networks in KO-ctrl cells compared to WT-ctrl cells. We also found that expression of wild type TOP1MT restored mitochondrial morphology in the Rescue cells, whereas the patient variants had an intermediate effect on mitochondrial morphology in the V1 and V2 cell lines.

\section{Discussion}

To investigate whether the compound heterozygous TOP1MT variants that we identified in a newborn patient with hypertrophic cardiomyopathy could impact protein function, we examined their ability to rescue several parameters in cells lacking TOP1MT. Our results reveal several significant differences between the rescue capabilities of both V1 and V2 TOP1MT variants compared to WT TOP1MT (summarized in Table 2). The fact that V1 and V2 variants could provide partial rescue in some conditions when re-expressed indicates that they retain some functionality. However, it should be noted that the variants were overexpressed $\sim 10$ fold above endogenous protein expression. Thus, any rescue is likely an overestimate of what would be expected at endogenous expression levels. Overall, our findings show that the V1 and V2 variants have impaired functionality. Intriguingly, our molecular characterization of the V1 and V2 variants also begins to provide novel mechanistic insight into the roles that TOP1MT plays in mediating mitochondrial functions. As detailed below, we noted that the V1 and V2 variants differed from each other with respect to their partial rescue for several of the parameters we investigated. These discrepancies suggest the V1 and V2 variants have distinct effects on the various TOP1MT functions.

\section{Nucleoid Size}

We found that loss of TOP1MT leads to smaller nucleoids. As each mtDNA nucleoid is estimated to contain $\sim 1.4$ copies of the mitochondrial genome (reflecting the ongoing replication of mtDNA molecules)[31], smaller nucleoid sizes likely do not reflect fewer copies of the mtDNA in each nucleoid, especially as we saw an increase in mtDNA copy number. We also showed that there were no deletions in the mtDNA, excluding another alternative explanation for smaller nucleoids. The finding of smaller nucleoids is consistent with previous work showing that loss of TOP1MT in MEF cells and murine tissues leads to increased mtDNA negative supercoiling [24]. Thus, we conclude that the smaller nucleoid sizes in TOP1MT KO cells reflect higher levels of negative supercoiling leading to more compact nucleoid structures. Both V1 and V2 variants were able to partially rescue the nucleoid size phenotype, with V1 having slightly stronger rescue, though neither was as efficient as the WT TOP1MT. This finding indicates that both V1 and V2 variants likely have reduced topoisomerase activity. 


\section{mtDNA Copy Number and Replication}

We also observed relevant differences in mtDNA copy number and replication. Consistent with a trend for increased numbers of nucleoids via confocal microscopy, an increase in mtDNA copy number in TOP1MT KO cells was quantified by PCR. Nonetheless, despite an increase in steady state mtDNA copy number, we found that TOP1MT KO cells had decreased rates of mtDNA replication during repletion following ethidium bromide treatment, as noted in previous work $[22,28]$. Curiously, while the V2 variant was able to restore steady state copy number and rescue mtDNA replication during repletion, the V1 variant showed no rescue at all in the context of mtDNA copy number or replication.

With respect to how TOP1MT impacts mtDNA copy number and replication, it is worth noting that although the human mtDNA has been studied for over four decades, there are still several unknowns about how it is regulated. Relevant to the current work, there is evidence that the mechanism of mtDNA replication varies depending on the specific conditions such as 'maintenance replication' to preserve copy number, compared to 'induced replication' to restore copy number following depletion[32]. Specifically, 'induced replication' appears to occur by reducing replication termination events on the $\mathrm{H}$-strand that otherwise would lead to D-loop formation[33]. Notably, decreased $\mathrm{H}$-strand termination also occurs under physiological conditions such as T lymphocytes undergoing rapid cell proliferation[34]. Critically, there is a cluster of TOP1MT binding sites in the D-loop region near the site where replication termination occurs[12]. Thus, the fact that TOP1MT KO cells have decreased recovery rates under 'induced' conditions suggests that TOP1MT is required to avoid $\mathrm{H}$-strand termination. Conversely, TOP1MT may also inhibit 'maintenance replication', given the increased steadystate levels observed in TOP1MT KO cells. Combined with the of ability of both V1 and V2 variants to partially rescue nucleoid size, the discrepancy in the ability of V1 and V2 to rescue mtDNA copy number and repletion suggests that the ability of TOP1MT to regulate these parameters may be independent of its topoisomerase activity. Alternatively, there could be a threshold effect with respect to the amount of supercoiling linked to mtDNA copy number and repletion, with the V1 and V2 variants on either side.

It is also interesting that while overexpression of WT TOP1MT significantly increased mtDNA repletion rates, it did not have a major impact on mtDNA copy number. Overall, these observations suggest that TOP1MT plays distinct roles in regulating mtDNA replication and maintaining copy number. Moreover, the differences between the V1 and V2 variants could be useful in further elucidating how TOP1MT impacts mtDNA replication.

\section{Transcription}

Another interesting finding was the fact that despite maintaining a higher steady-state mIDNA copy number, TOP1MT KO cells have reduced expression of mitochondrial encoded mRNA genes. With respect to rescue, the V1 variant was able to restore transcript levels to a similar degree as the WT protein, while the V2 variant only had partial rescue capabilities. The similar rescue trends with respect to transcription and nucleoid size, with V1 providing better rescue than $\mathrm{V} 2$, suggest that TOP1MT affects transcription via its topoisomerase activity. This finding is consistent with previous reports that DNA topology impacts mitochondrial transcription in vitro[35-39], and that TOP1MT appears to regulate transcription via its 
supercoiling activity[14, 29]. Notably, our findings also suggest that a partial rescue in supercoiling activity is sufficient to rescue transcription.

Our findings that TOP1MT KO cells had reduced transcript levels contrast with previous reports that TOP1MT inhibits transcription and that KO cells had elevated transcript levels[14, 29]. The reasons for this discrepancy are likely due to differences in growth conditions, as many different parameters can impact mitochondrial function[40]. Our data suggest that different seeding densities are critical for determining how TOP1MT impacts transcript levels. Future work will need to look at how the other roles of TOP1MT are impacted by cell density. Indeed, the cell density could impact growth rate, which would be relevant to the different modes of mtDNA replication and D-loop formation discussed above. Regardless of the specific growth conditions, it is evident that TOP1MT clearly affects mitochondrial transcript levels, likely via changes in supercoiling.

\section{Mitochondrial Proteins and Respiration}

We observed decreased protein levels of mtDNA-encoded OxPhos proteins in TOP1MT KO cells. A similar trend in reduced expression of nuclear-encoded OxPhos proteins was also observed, and likely reflects the fact that expression of the mitochondrial encoded subunits is rate limiting. The overall reduction in OxPhos proteins most likely explains the reduced oxygen consumption and mitochondrial impairments in TOP1MT KO cells. Although the reduced transcript levels that we observed in TOP1MT KO cells could explain the reduced translation of mtDNA-encoded genes, this conclusion is complicated by the fact that TOP1MT also has a distinct role in mediating mitochondrial translation by directly interacting with the ribosome[15]. In this regard, the differences in partial rescue of different TOP1MT functions by V1 and V2 provides unique insight, as neither V1 or V2 re-expression was sufficient to restore translation or oxygen consumption. However, given that V1 and V2 were able to restore transcript levels fully and partially, respectively, but neither fully rescued protein levels, we suggest that impaired translation in the V1 and V2 lines is largely responsible for the decreased mitochondrial function in these cells. Though little is known about exactly how TOP1MT regulates translation, our findings suggest that the role TOP1MT plays in translation is independent of its topoisomerase activity and changes in transcripts. Moreover, it is likely that the role TOP1MT plays in translation is also independent of its roles in meditating mtDNA replication. Finally, the V1 and V2 variants, which both have deficient translation capabilities, could prove valuable tools in future studies looking at how TOP1MT regulates mitochondrial translation.

\section{Mitochondrial Morphology}

We observed and quantified an increase in mitochondrial hyperfusion in TOP1MT KO cells, consistent with previous reports in TOP1MT knockout mouse embryonic fibroblasts[41]. Although fragmentation of mitochondrial networks is typically associated with mitochondrial dysfunction, hyperfusion of the mitochondrial network can also occur in response to various stresses[42]. While overexpression of wild type TOP1MT restored mitochondrial morphology, V1 and V2 variants were capable of partially reverting the mitochondrial morphology. This rescue pattern resembles what we observed with respect to changes in mitochondrial nucleoid size, suggesting that changes in mtDNA nucleoid topology can promote mitochondrial 
hyperfusion. Although the mechanism through which changes in mtDNA might impact the mitochondrial network is unknown, it is notable that a similar hyperfusion of the mitochondrial network occurs in cells with heterozygous loss of TFAM, a key mtDNA packaging protein and transcription factor[43].

\section{TOP1MT in Human Disease}

Although the TOP1MT gene is not formally recognized as a human disease gene, our novel findings, combined with previous work by others, strengthen the argument that TOP1MT should be considered for its role in human disease. In a study of 40 patients with disorders of mtDNA maintenance, which sequenced 39 candidate genes linked to mtDNA maintenance, two predicted pathogenic heterozygous variants in TOP1MT were identified[44]. However, given the limited number of genes sequenced at the time, and a lack of mechanistic studies, a direct correlation between the patient phenotype and pathogenicity of the candidate variants was not established. In a separate study, 30 potentially deleterious TOP1MT variants occurring in the normal population were reported[23], including the V2 variant (R338L) that we studied here. Again, however, no functional characterization of these variants was performed. Meanwhile, a functional study of TOP1MT single nucleotide polymorphisms (SNPs), found that some SNPs have reduced DNA relaxation activities in vitro[45]. Notably, these SNPs include a rare variant (E168G) found in a patient with mitochondrial deficiency syndrome, and two common single nucleotide variants (V256I and R525W) that are most often mutually exclusive, suggesting there is selection against both variants being present together.

Mouse studies also support a role for TOP1MT in maintaining mitochondrial function. Although TOP1MT knockout mice are viable and fertile[13], they are sensitive to doxorubicininduced cardiotoxicity[23], have impaired liver regeneration [22], and embryonic fibroblasts from these mice have defective mitochondrial function[41]. Our findings are certainly consistent with the notion that the V1 and V2 variants contribute to mitochondrial dysfunction, most likely through some combination of impaired topoisomerase activity, altered mtDNA replication, and reduced mitochondrial translation. However, we cannot definitively ascribe the cardiomyopathy in our patient directly to mitochondrial dysfunction due to the V1 and V2. Nonetheless, the increased cardiotoxicity of TOP1MT KO mice to doxorubicin emphasizes the importance of TOP1MT to cardiac function. Additionally, mtDNA genomes in cardiac tissue are organized into complex catenated networks[46], which may make them more dependent on topoisomerase activity. Meanwhile, there is a significant transition in cardiac function at birth that has critical implications for mitochondrial function, as the heart adapts to changes in oxygen levels, increased energy demand, and a metabolic shift from glycolysis to betaoxidation. Thus, we suggest that impaired TOP1mt activity, combined with the dependence of the heart on mitochondrial function, is likely to be contributing to the cardiomyopathy in the patient.

\section{Conclusions}

Overall, our characterization of the V1 and V2 variants support the argument that TOP1MT should be considered as a mitochondrial disease gene. Additionally, characterization of our stable cells lines provides insight into the multiple distinct and interrelated functions of 
TOP1MT including relaxing mtDNA supercoiling, regulating mtDNA transcript levels, mediating mitochondrial replication, and promoting mitochondrial protein translation. By comparing the distinct rescue patterns of the V1 and V2 variants, our findings support that idea that the topoisomerase activity of TOP1MT is related to its role in regulating mitochondrial transcription, as well as changes in mitochondrial morphology. Meanwhile, the roles that TOP1MT plays in mtDNA replication, maintenance of mtDNA copy number, and mitochondrial translation appear to be separate from each other, as well as independent of the topoisomerase activity. Future characterization of the V1 and V2 variants, as well as other TOP1MT variants, will likely contribute to delineating the roles that TOP1MT plays in maintaining mitochondrial function and its role in human disease.

\section{Data Availability}

The data that support the findings of this work are available from the corresponding author upon request.

\section{Supplementary Data}

Supplementary Data are available at NAR Online.

\section{Funding}

This work was supported by funds provided by the Alberta Children's Hospital Research Institute (ACHRI), the Alberta Children's Hospital Foundation (ACHF) and the Natural Sciences and Engineering Research Council of Canada (NSERC - RGPIN-2016-04083) (T.S.). This work was also supported by the NIH Intramural Program, the Center for Cancer Research, US National Cancer Institute (BC006161 to Y.P., H.Z and S.H.). I.A.K. is a recipient of an ACHRI Graduate Studentship and William H. Davies scholarship. A.K. received research funding from MitoCanada. The funding sources were not involved in the study design, data collection and analysis, the writing of the report, nor in the decision to submit.

Conflict of interest statement. The authors declare that they have no conflict of interest.

Author contributions: I.A.K. conceived the study, designed, performed and analyzed experiments and wrote the manuscript. M.K. identified the TOP1MT patient variants. H.Z., S.H. and Y.P. generated the HCT116 TOP1MT KO cells, shared the TOP1MT cDNA, and provided input on the project. A.K. performed the clinical workup of the patient, provided input on the project, and edited the manuscript. T.S. conceived and designed the study, analyzed experiments, supervised the study, and wrote the manuscript. 


\section{References}

1. Kukat, C., et al., Super-resolution microscopy reveals that mammalian mitochondrial nucleoids have a uniform size and frequently contain a single copy of mtDNA. Proc Natl Acad Sci U S A, 2011. 108(33): p. 13534-9.

2. Al Khatib, I. and T.E. Shutt, Advances Towards Therapeutic Approaches for mtDNA Disease. Adv Exp Med Biol, 2019. 1158: p. 217-246.

3. Nicholls, T.J. and M. Minczuk, In D-loop: 40 years of mitochondrial 75 DNA. Exp Gerontol, 2014. 56: p. 175-81.

4. Chang, D.D. and D.A. Clayton, Priming of human mitochondrial DNA replication occurs at the light-strand promoter. Proc Natl Acad Sci U S A, 1985. 82(2): p. 351-5.

5. Bonawitz, N.D., D.A. Clayton, and G.S. Shadel, Initiation and beyond: multiple functions of the human mitochondrial transcription machinery. Mol Cell, 2006. 24(6): p. 813-25.

6. Shutt, T.E., et al., Core human mitochondrial transcription apparatus is a regulated twocomponent system in vitro. Proc Natl Acad Sci U S A, 2010. 107(27): p. 12133-8.

7. Shutt, T.E. and M.W. Gray, Twinkle, the mitochondrial replicative DNA helicase, is widespread in the eukaryotic radiation and may also be the mitochondrial DNA primase in most eukaryotes. J Mol Evol, 2006. 62(5): p. 588-99.

8. Korhonen, J.A., et al., Reconstitution of a minimal mtDNA replisome in vitro. Embo j, 2004. 23(12): p. 2423-9.

9. Sobek, S. and F. Boege, DNA topoisomerases in mtDNA maintenance and ageing. Exp Gerontol, 2014. 56: p. 135-41.

10. Zhang, H., et al., Human mitochondrial topoisomerase I. Proc Natl Acad Sci U S A, 2001. 98(19): p. 10608-13.

11. Zhang, X., et al., Redox-induced apoptosis of human oocytes in resting follicles in vitro. J Soc Gynecol Investig, 2006. 13(6): p. 451-8.

12. Zhang, $\mathrm{H}$. and Y. Pommier, Mitochondrial topoisomerase I sites in the regulatory D-loop region of mitochondrial DNA. Biochemistry, 2008. 47(43): p. 11196-203.

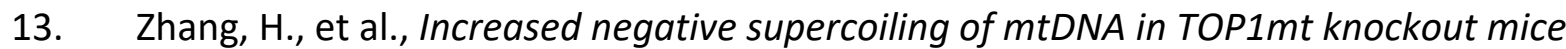
and presence of topoisomerases $\| \alpha$ and $\| B$ in vertebrate mitochondria. Nucleic Acids Res, 2014. 42(11): p. 7259-67.

14. Sobek, S., et al., Negative regulation of mitochondrial transcription by mitochondrial topoisomerase I. Nucleic Acids Res, 2013. 41(21): p. 9848-57.

15. Baechler, S.A., et al., The mitochondrial type IB topoisomerase drives mitochondrial translation and carcinogenesis. Nat Commun, 2019. 10(1): p. 83.

16. Kerr, M., et al., MITO-FIND: A study in 390 patients to determine a diagnostic strategy for mitochondrial disease. Mol Genet Metab, 2020. 131(1-2): p. 66-82.

17. Sabouny, R., et al., Characterization of the C584R variant in the mtDNA depletion syndrome gene FBXL4, reveals a novel role for FBXL4 as a regulator of mitochondrial fusion. Biochim Biophys Acta Mol Basis Dis, 2019. 1865(11): p. 165536.

18. Donkervoort, S., et al., MSTO1 mutations cause mtDNA depletion, manifesting as muscular dystrophy with cerebellar involvement. Acta Neuropathol, 2019. 138(6): p. 1013-1031. 
19. Sharma, G., et al., Characterization of a novel variant in the HR1 domain of \&lt;em\&gt;MFN2\&lt;/em\&gt; in a patient with ataxia, optic atrophy and sensorineural hearing loss. bioRxiv, 2021: p. 2021.01.11.426268.

20. Nishigaki, Y., R. Marti, and M. Hirano, ND5 is a hot-spot for multiple atypical mitochondrial DNA deletions in mitochondrial neurogastrointestinal encephalomyopathy. Hum Mol Genet, 2004. 13(1): p. 91-101.

21. Eaton, J.S., et al., Ataxia-telangiectasia mutated kinase regulates ribonucleotide reductase and mitochondrial homeostasis. J Clin Invest, 2007. 117(9): p. 2723-34.

22. Khiati, S., et al., Lack of mitochondrial topoisomerase I (TOP1mt) impairs liver regeneration. Proc Natl Acad Sci U S A, 2015. 112(36): p. 11282-7.

23. Khiati, S., et al., Mitochondrial topoisomerase I (top1mt) is a novel limiting factor of doxorubicin cardiotoxicity. Clin Cancer Res, 2014. 20(18): p. 4873-81.

24. Zhang, $\mathrm{H}$., et al., Increased negative supercoiling of mtDNA in TOP1mt knockout mice and presence of topoisomerases I/alpha and Ilbeta in vertebrate mitochondria. Nucleic Acids Res, 2014. 42(11): p. 7259-67.

25. Yu, M., et al., Depletion of mitochondrial DNA by ethidium bromide treatment inhibits the proliferation and tumorigenesis of T47D human breast cancer cells. Toxicol Lett, 2007. 170(1): p. 83-93.

26. Kao, L.P., D. Ovchinnikov, and E. Wolvetang, The effect of ethidium bromide and chloramphenicol on mitochondrial biogenesis in primary human fibroblasts. Toxicol Appl Pharmacol, 2012. 261(1): p. 42-9.

27. Moraes, C.T., L. Kenyon, and H. Hao, Mechanisms of human mitochondrial DNA maintenance: the determining role of primary sequence and length over function. Mol Biol Cell, 1999. 10(10): p. 3345-56.

28. Baechler, S.A., et al., Beyond the unwinding: role of TOP1MT in mitochondrial translation. Cell Cycle, 2019. 18(19): p. 2377-2384.

29. Dalla Rosa, I., et al., Transcription profiling suggests that mitochondrial topoisomerase IB acts as a topological barrier and regulator of mitochondrial DNA transcription. J Biol Chem, 2017. 292(49): p. 20162-20172.

30. Goffart, S., A. Hangas, and J.L.O. Pohjoismaki, Twist and Turn-Topoisomerase Functions in Mitochondrial DNA Maintenance. Int J Mol Sci, 2019. 20(8).

31. Kukat, C., et al., Super-resolution microscopy reveals that mammalian mitochondrial nucleoids have a uniform size and frequently contain a single copy of mtDNA. Proc Natl Acad Sci U S A, 2011. 108(33): p. 13534-9.

32. Fish, J., N. Raule, and G. Attardi, Discovery of a major D-loop replication origin reveals two modes of human mtDNA synthesis. Science, 2004. 306(5704): p. 2098-101.

33. Brown, T.A. and D.A. Clayton, Release of replication termination controls mitochondrial DNA copy number after depletion with 2',3'-dideoxycytidine. Nucleic Acids Res, 2002. 30(9): p. 2004-10.

34. Kai, Y., et al., Mitochondrial DNA replication in human T lymphocytes is regulated primarily at the $H$-strand termination site. Biochim Biophys Acta, 1999. 1446(1-2): $p$. 126-34.

35. Yaginuma, K., et al., A new RNA polymerase and in vitro transcription of the origin of replication from rat mitochondrial DNA. Nucleic Acids Res, 1982. 10(23): p. 7531-42. 
36. Buzan, J.M. and R.L. Low, Preference of human mitochondrial RNA polymerase for superhelical templates with mitochondrial promoters. Biochem Biophys Res Commun, 1988. 152(1): p. 22-9.

37. Fukuoh, A., et al., DNA conformation-dependent activities of human mitochondrial RNA polymerase. Genes Cells, 2009. 14(8): p. 1029-42.

38. Barat-Gueride, M., C. Dufresne, and D. Rickwood, Effect of DNA conformation on the transcription of mitochondrial DNA. Eur J Biochem, 1989. 183(2): p. 297-302.

39. Zollo, O. and N. Sondheimer, Topological requirements of the mitochondrial heavystrand promoters. Transcription, 2017. 8(5): p. 307-312.

40. Bayona-Bafaluy, M.P., J. Montoya, and E. Ruiz-Pesini, Oxidative phosphorylation system and cell culture media. Trends Cell Biol, 2021.

41. Douarre, C., et al., Mitochondrial topoisomerase I is critical for mitochondrial integrity and cellular energy metabolism. PLoS One, 2012. 7(7): p. e41094.

42. Tondera, D., et al., SLP-2 is required for stress-induced mitochondrial hyperfusion. Embo j, 2009. 28(11): p. 1589-600.

43. West, A.P., et al., Mitochondrial DNA stress primes the antiviral innate immune response. Nature, 2015. 520(7548): p. 553-7.

44. Wang, W., et al., Identification of rare DNA variants in mitochondrial disorders with improved array-based sequencing. Nucleic Acids Res, 2011. 39(1): p. 44-58.

45. Zhang, H., et al., Distribution bias and biochemical characterization of TOP1MT single nucleotide variants. Sci Rep, 2017. 7(1): p. 8614.

46. Pohjoismäki, J.L., et al., Human heart mitochondrial DNA is organized in complex catenated networks containing abundant four-way junctions and replication forks. J Biol Chem, 2009. 284(32): p. 21446-57. 
Figure 1. Generation of stable lines expressing wild-type (WT) or mutant forms of TOP1MT, as well as control lines for both WT and KO cells.

a)

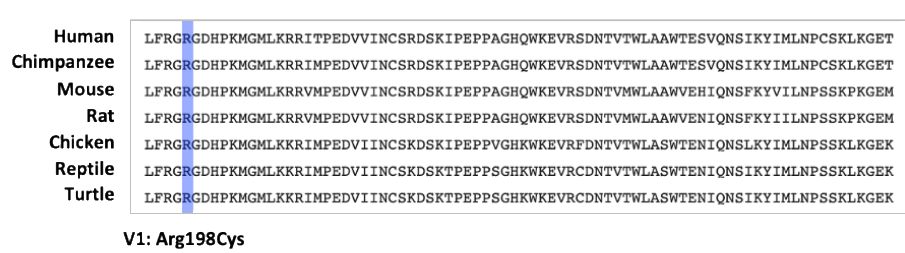

Human Chimpanzee

Mouse

Rat

Chicken

Turtle b)

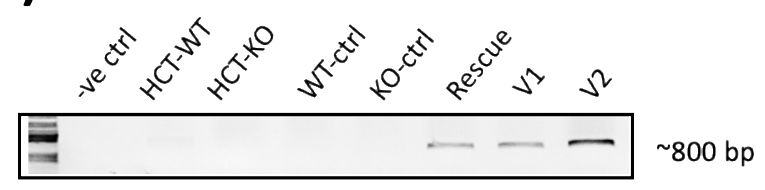

d)

c)
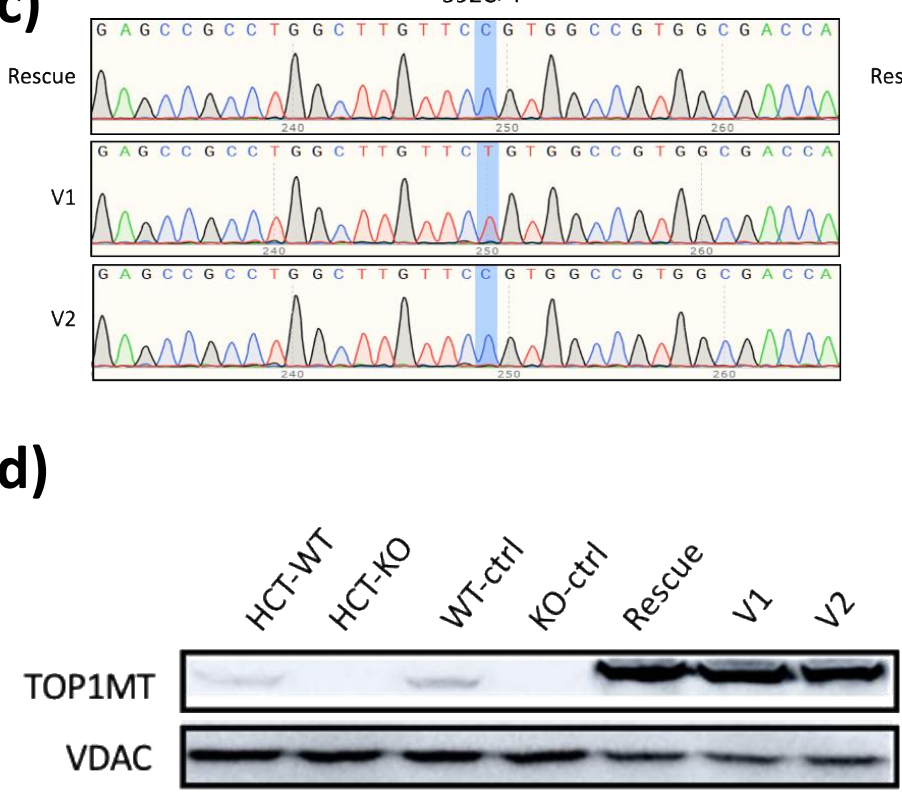

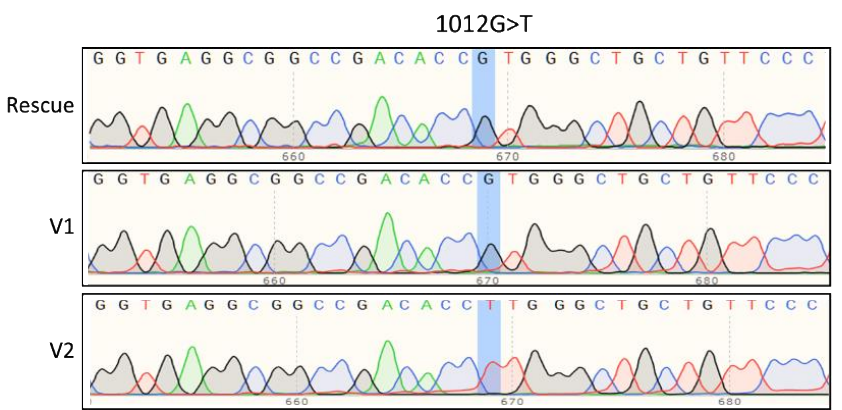

e)

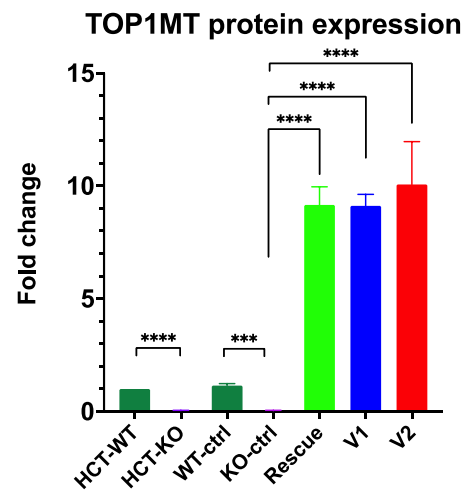

Figure 1. Generation of stable lines expressing wild-type (WT) or mutant forms of TOP1MT, as well as control lines for both WT and KO cells. a) Amino acid alignments of TOP1MT proteins from a variety of vertebrate species generated using COBALT shows the conservation of the amino-acids variants identified in the patient. b) PCR amplification targeting the coding region of the viral-encoded TOP1MT open reading frame, spanning patient variants, confirms amplicons are present only in HCT-KO cell lines stably re-expressing TOP1MT, namely the Rescue, V1 and V2 lines. c) Chromatograms from Sanger sequencing of the amplicons produced from (b), for the $592 \mathrm{C}$ and $1012 \mathrm{G}$ residues (shaded), confirms the identity of the cell lines. d) Western blots probed for TOP1MT antibody confirms the HCT-KO line lacks TOP1MT and reveals TOP1MT expression in stably transduced cell lines. VDAC was probed as a load control. e) Quantification of western blots from 4 replicates show the fold change in protein abundance compared to VDAC control from four different replicates. All statistical analysis were done using unpaired student $t$-test; where error bars represent standard error of mean and $p$ values $* * *<0.001$ and $* * * *<0.0001$. 
Figure 2. Representative confocal images of TOP1MT localization in stable lines.

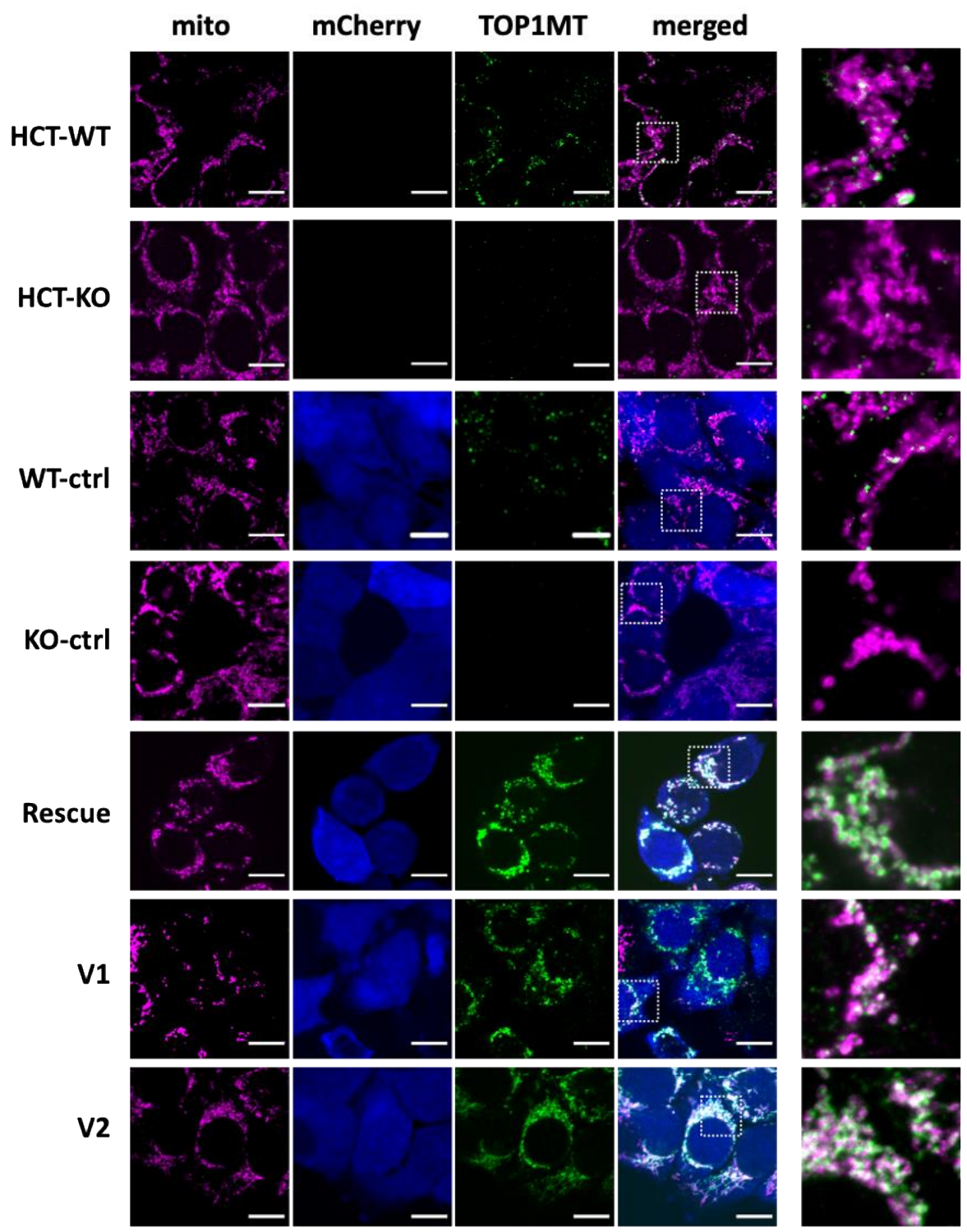

Figure 2. Representative confocal images of TOP1MT localization in stable lines. Fixed Cells were stained for immunofluorescence with anti-TOMM20 (mitochondria, magenta) and antiTOP1MT (green) primary antibodies. Viral transduction was confirmed by expression of mCherry (Blue). The final column shows the zoomed-inn region indicated in the merged column. Images confirm lack of TOP1MT in HCT-KO cells and show the mitochondrial localization of TOP1MT-WT and both V1 and V2 patient variants. Scale bar represent $10 \mu \mathrm{m}$. 
Figure 3. Analysis of mitochondrial nucleoids and mtDNA deletions in control and TOP1MT-KO cells.

a)
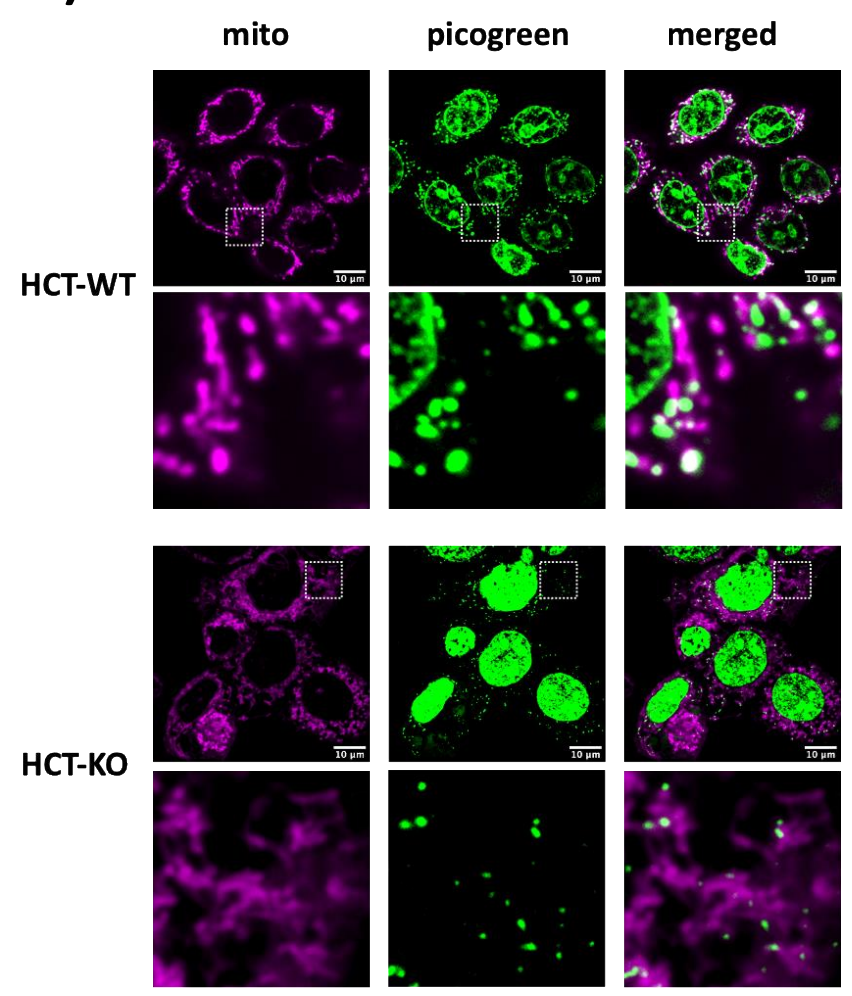
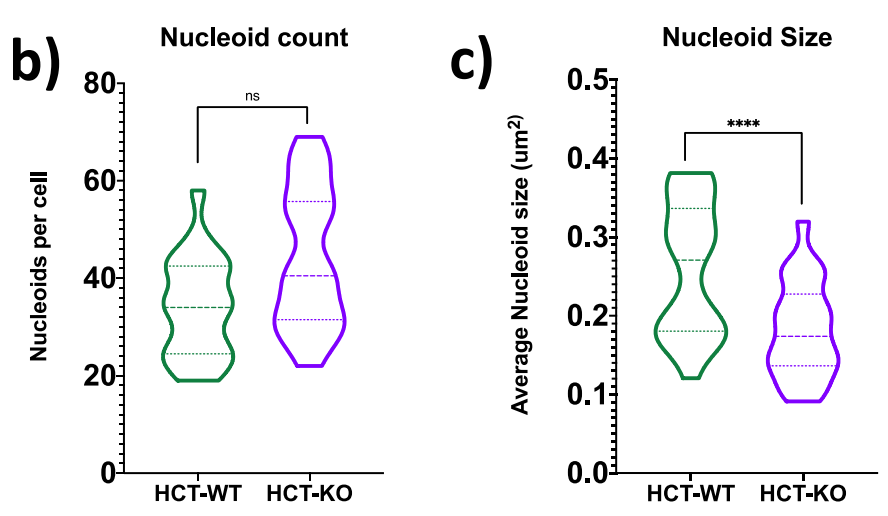

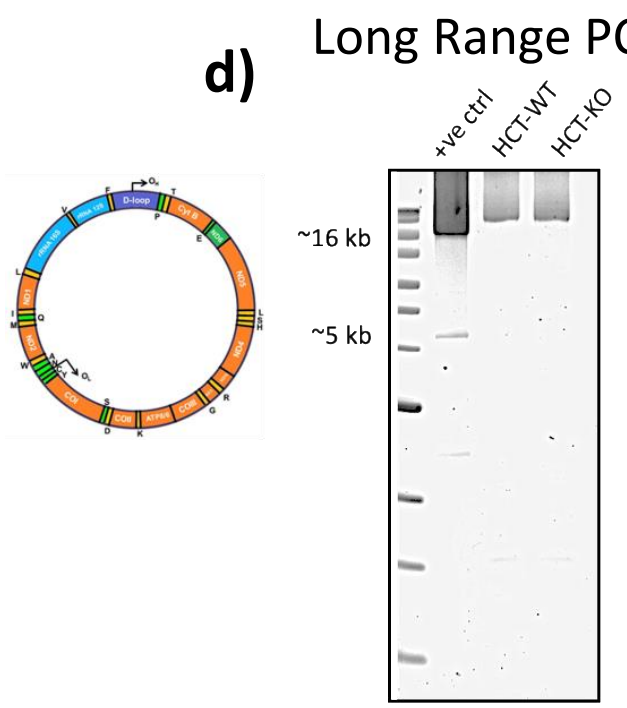

Figure 3. Analysis of mitochondrial nucleoids and mtDNA deletions in control and TOP1MT-KO cells. a) Representative confocal images of live cells stained with MitoTracker Far Red (mitochondria) and PicoGreen (dsDNA: nuclear and mtDNA) where the final column shows the merged image between mitochondria and dsDNA. Scalebars represent $10 \mu \mathrm{m}$. A zoomed-inn region is shown on the lower row corresponding to the represented image above for both HCT-WT and HCT-KO cells. Violin plots showing quantification of mtDNA nucleoids from 25 cells stained as in (a), represented as nucleoid count per cell (b) and average nucleoid size per cell (c) P-values were determined by a Kolmogorov-Smirnov test for all measured nucleoids, where ' $n s$ ' signifies no significant differences between indicated groups and $* * * *<0.0001$. d) Long range PCR of mtDNA in HCT-WT and KO cells show only the full-length $16.3 \mathrm{~kb}$ product, indicating no mtDNA deletions are detectable. DNA form a cell harbouring a $5 \mathrm{~kb}$ deletion was used as a +ve control to show the protocol detects deletions. 
Figure 4. Nucleoid sizes in TOP1MT rescue cell lines.

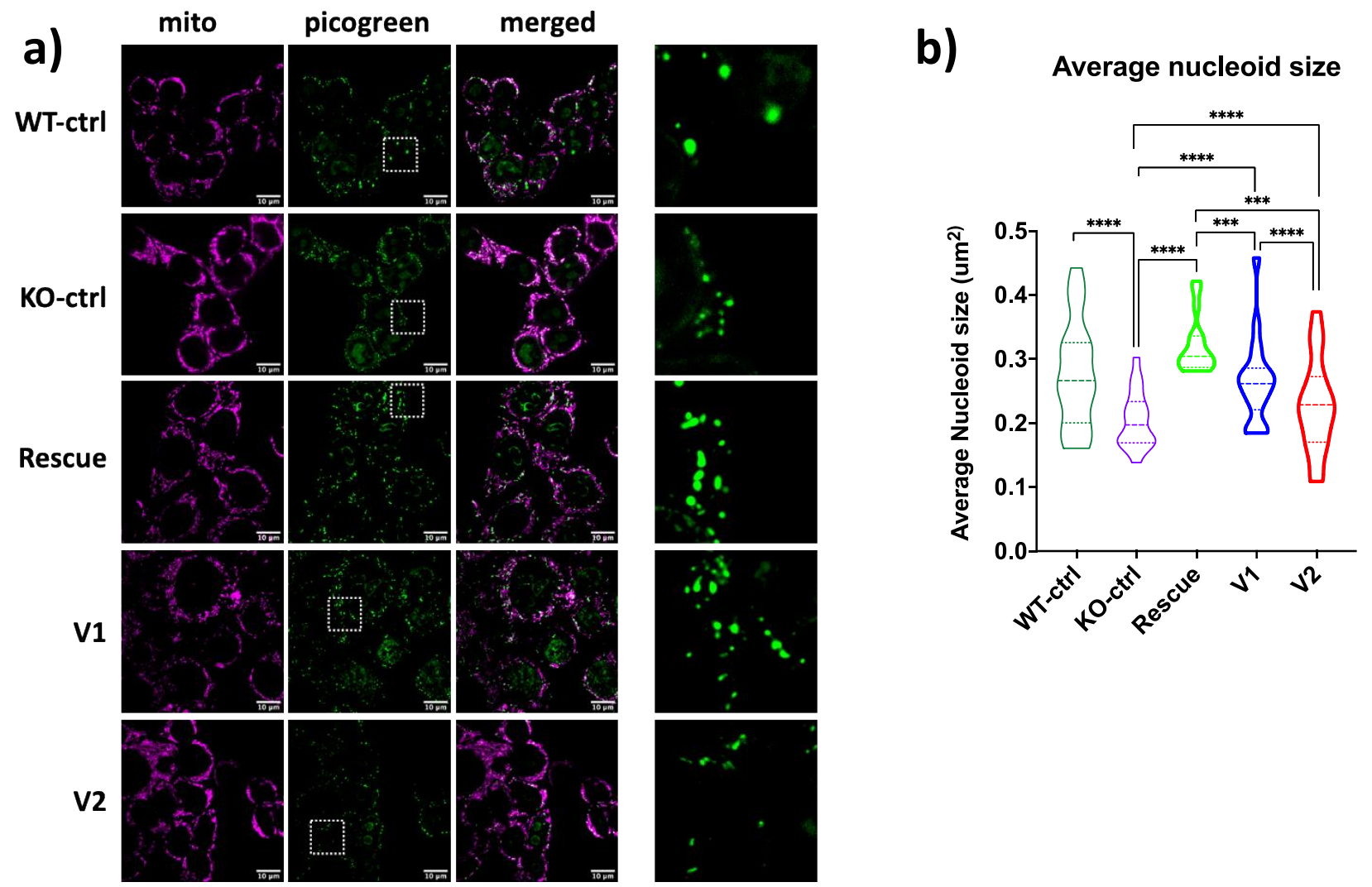

Figure 4. Nucleoid sizes in TOP1MT rescue cell lines. a) Representative confocal images stained of live cells with MitoTracker Far Red (mitochondria) and PicoGreen (dsDNA: mtDNA nucleoids and nucleus). Merged column represents the combined image of mitochondria and dsDNA where the final column shows the zoomed-inn region of nucleoids for size comparison. Scalebars represent 10 $\mu \mathrm{m}$. b) Quantification of mtDNA nucleoid sizes in cells stained as in (a). Data represents average nucleoid sizes from at least 15 cells for each group. P-values were determined by a KolmogorovSmirnov test for all measured nucleoids, with $* * *<0.001$ and $* * * *<0.0001$. 
Figure 5. mtDNA replication and expression in TOP1MT rescue cell lines.

a) mtDNA copy number b)

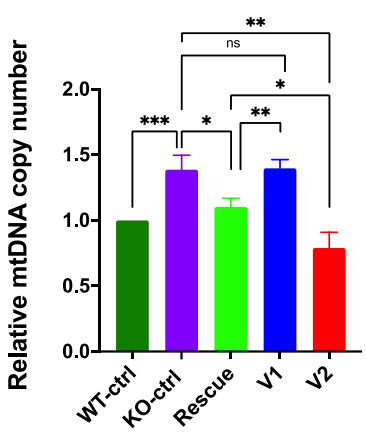

d)

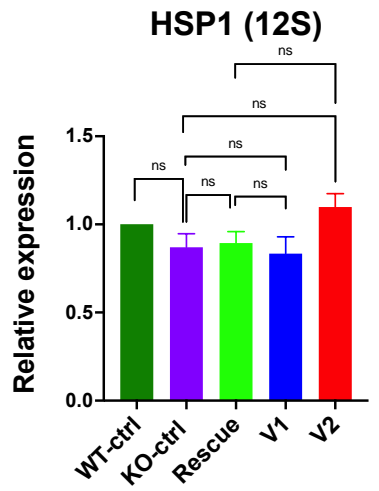

b) mtDNA copy number recovery

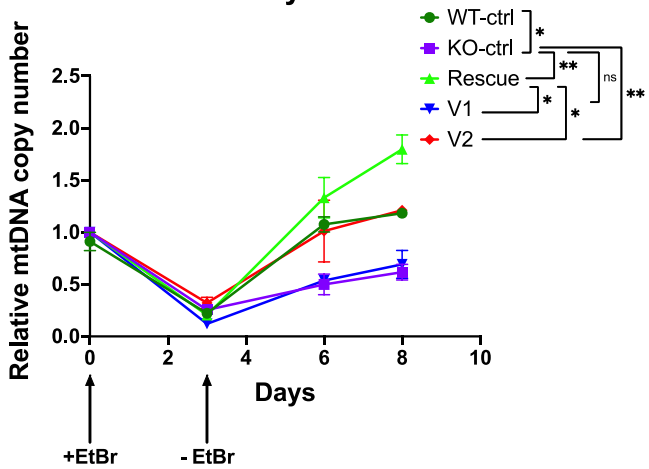

C)

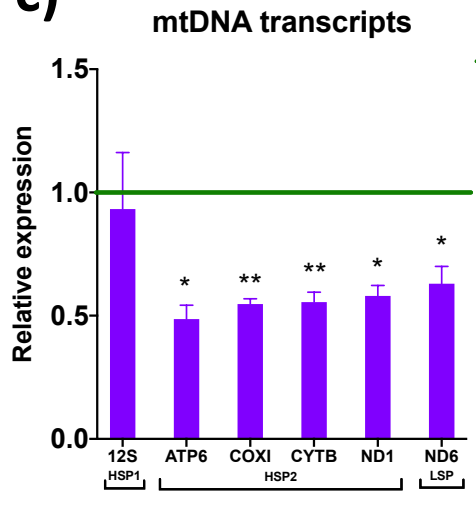

- HCT-WT

HCT-KO

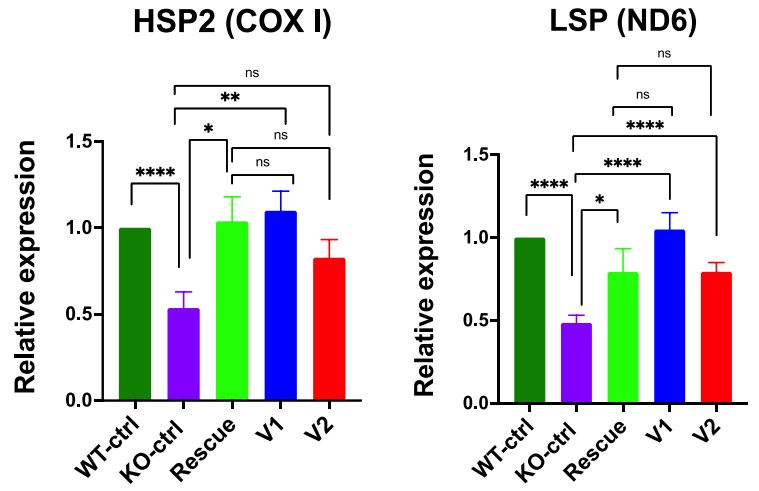

Figure 5. mtDNA replication and expression in TOP1MT rescue cell lines. Data represents at least three independent biological replicates. a) Copy number of the mtDNA, normalized to $18 \mathrm{~S}$, obtained via quantitative PCR. b) Recovery of mtDNA copy number, as determined in a), following three days depletion with EtBr. c) Quantification of mtDNA transcripts in HCT-WT and HCT-KO cell lines via quantitative RT-PCR for the indicated genes from the three mtDNA promoters: HSP1, LSP and HSP2. (d) Quantification of mtDNA transcripts in stable TOP1mt rescue lines for HSP1 (12S), HSP2 (COX I) and LSP (ND6). Error bars represent standard error of mean. P-values were determined by unpaired student t-test such that $*<0.05, * *<0.01, * * *<0.001$ and ${ }^{* * * *}<0.0001$. ' $\mathrm{ns}$ ' signifies no significant differences between indicated groups. 
Figure 6. Mitochondrial protein expression and oxygen consumption in TOP1MT rescue lines.

a)

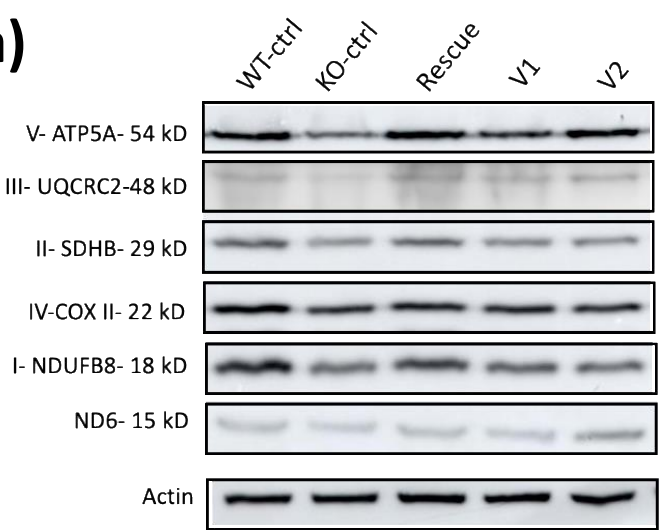

b)

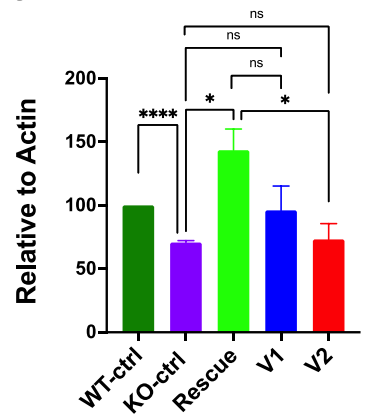

f)

i)

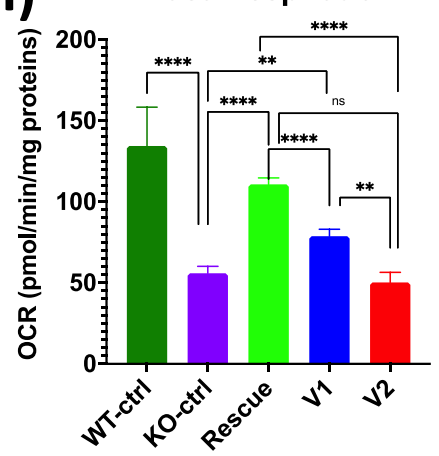

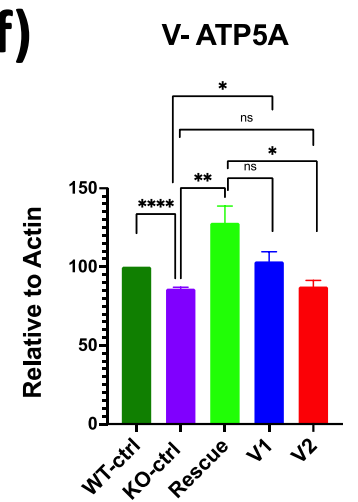

Basal respiration h)

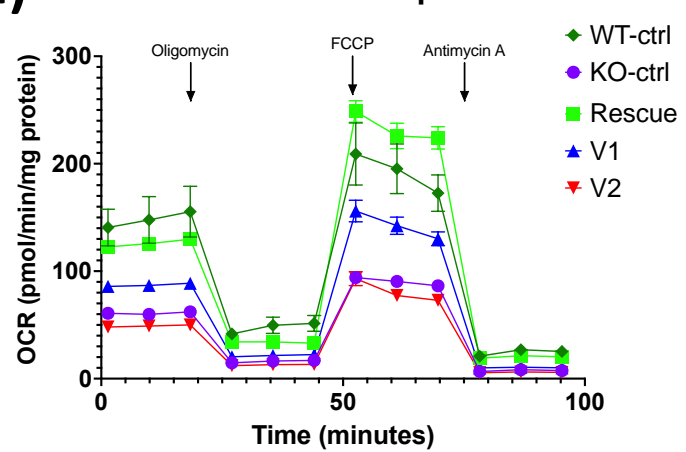

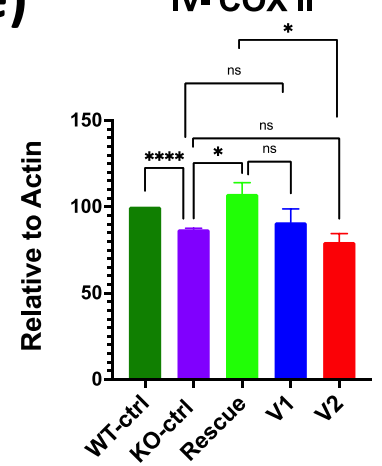

I- NDUFB8

c)

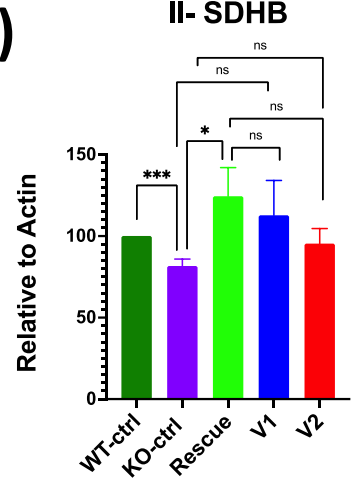

d)

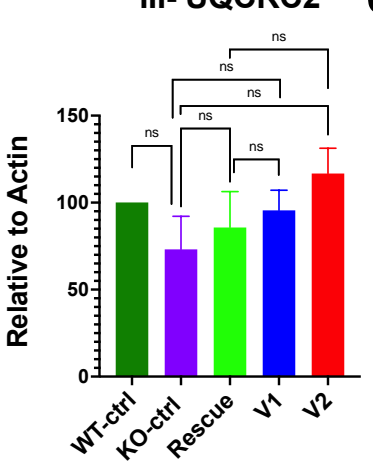

Figure 6. Mitochondrial protein expression and oxygen consumption in TOP1MT rescue lines. a) Representative western blots of the following proteins from different OXPHOS complexes: NDUFB8 (Complex I), SDHB (Complex II), UQCRC2 (Complex III), COXII (Complex IV), ATP5A (Complex V) and ND6 (Complex I). Quantification from at least three independent biological replicates of protein levels determined by western blot as in a) for: NDUFB8 (b), SDHB (c), UQCRC2 (d), COXII (e), ATP5A (f) and ND6 (g). Expression was normalized to expression the Actin load control. h) Oxygen consumption rates (OCR) over time analyzed in the indicated cell lines using the Seahorse XF24 extracellular flux analyzer. The OCR data was used to calculate basal respiration (i), maximal respiration (j) and ATP production (k). Data presented is from two independent biological replicates each with 5 technical replicates. Error bars represent standard error of mean. P-values were determined by an unpaired student t-test with $*<0.05, * *<0.01, * * *<0.001$ and $* * * *<0.0001$. 'ns' signifies no significant differences between indicated groups. 
Figure 7. Mitochondrial network morphology in TOP1MT lines.

a)
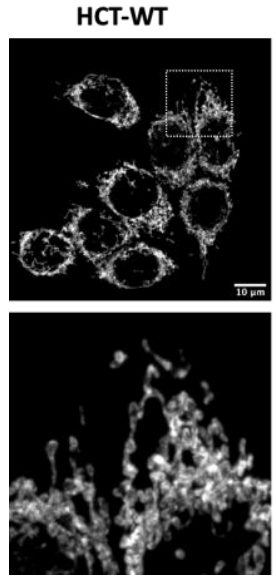

d)
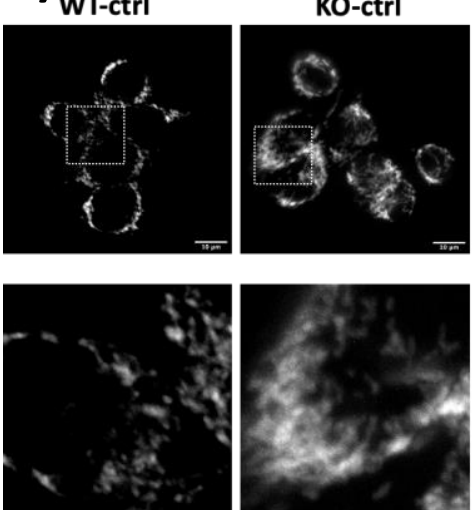

HCT-KO
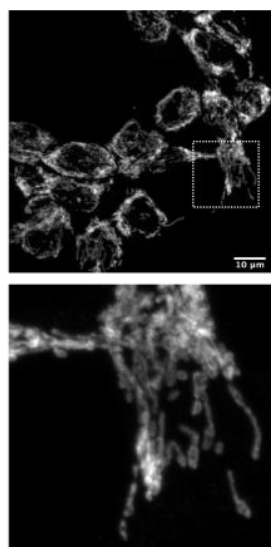

b)

Mitochondrial morpholgy

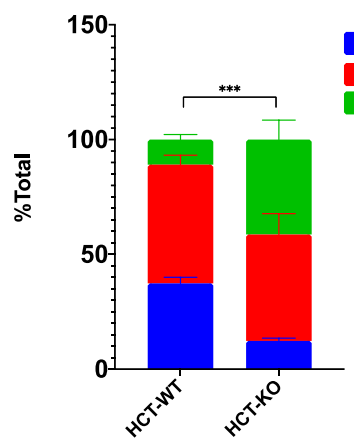

c)

Average mitochondrial length

Fragmented
Intermediate
Fused

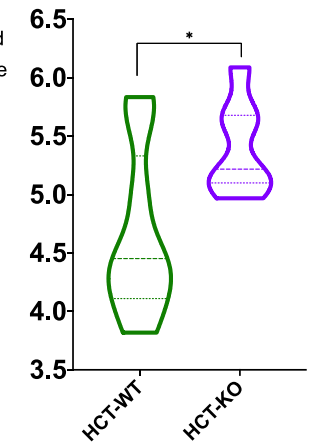

e)

V1
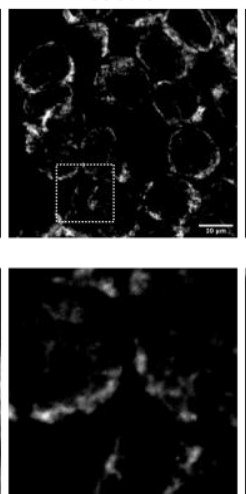
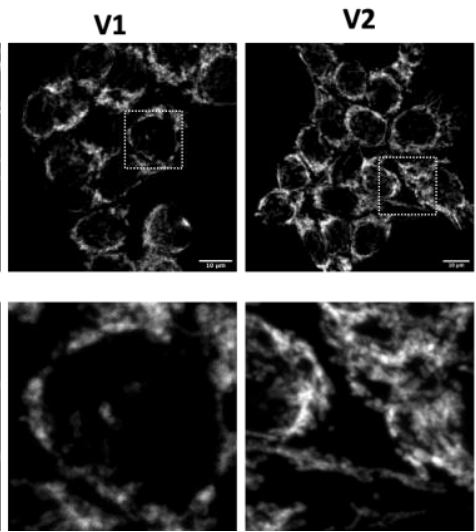

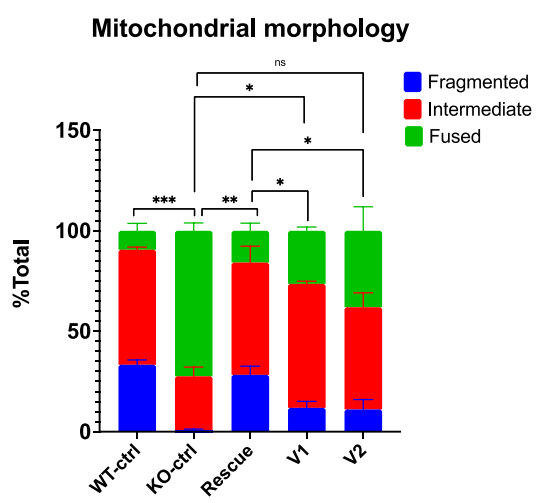

Figure 7. Mitochondrial network morphology in TOP1MT lines. a,d) Representative confocal images of mitochondrial networks from fixed cells and stained for immunofluorescence with the outer membrane protein TOMM20. Scalebars are $10 \mu \mathrm{m}$. b,e) Qualitative quantification of mitochondrial morphology for cells stained as in (a) and (e), respectively. Analysis for each line was performed on three technical replicates of at least 50 cells each. c) Quantitative analysis of average mitochondrial length from 20 cells as in (a), determined using ImageJ, confirms the qualitative analysis in (b). Error bars represent standard error of mean. P-values were determined by an

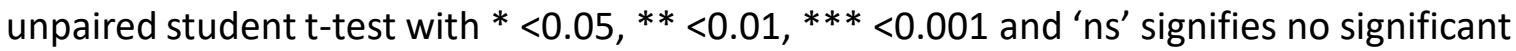
differences between indicated groups. 


\begin{tabular}{|c|c|c|c|c|}
\cline { 2 - 5 } & KO-ctrl & Rescue & V1 & V2 \\
\hline Nucleoid size & Smaller & +++ & ++ & + \\
\hline mtDNA Copy number & Higher & +++ & - & +++ \\
\hline mtDNA Repletion & Slower & +++ & - & ++ \\
\hline Mitochondrial Transcripts & Lower & +++ & +++ & ++ \\
\hline OXPHOS Proteins & Lower & +++ & - & - \\
\hline Mitochondrial Oxygen consumption & Lower & +++ & + & + \\
\hline Mitochondrial morphology & Fused & +++ & ++ & + \\
\hline
\end{tabular}

Table 2. Summary of analyzed phenotypes. Analyzed phenotypes where +++ represents full rescue, ++ represent moderate rescue, + for weak rescue and - for no rescue at all. 